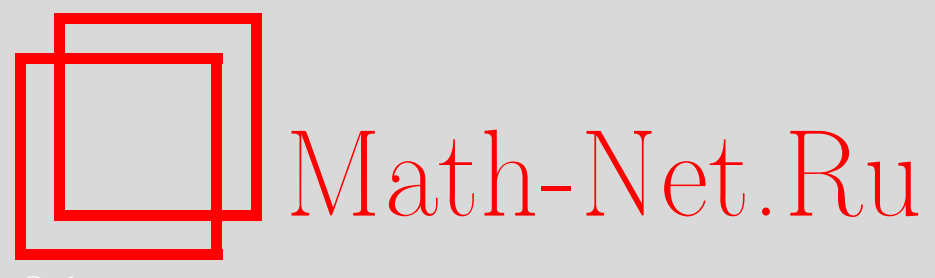

А. Райгородский, Одна задача о раскраске, Квант, 2019, номер 8, 15-22

DOI: https://doi.org/10.4213/kvant20190802

Использование Общероссийского математического портала Math-Net.Ru подразумевает, что вы прочитали и согласны с пользовательским соглашением http://www.mathnet.ru/rus/agreement

Параметры загрузки:

IP : 54.174 .149 .18

26 апреля 2023 г., $11: 51: 48$

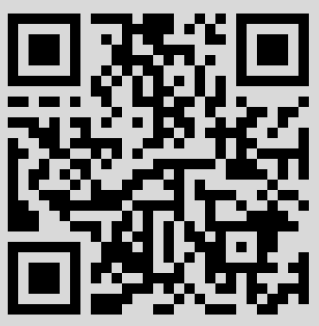




\section{Одна задача о раскраске}

\section{А.РАЙГОРОДСКИЙ}

\section{Задача для затравки}

Для затравки рассмотрим следующую задачу. В классе учатся 30 человек. Из них отбираются 5 лучших комбинаторщиков, 5 лучших числовиков, 5 лучших вероятностников и так далее. Всего 15 предметов. Конечно, эти пятерки лучших могут как угодно пересекаться: заранее мы не знаем, кто окажется сильнее в каком из предметов. Вопрос: всегда ли можно так рассадить наших 30 школьников по двум кабинетам, чтобы в каждом кабинете был хотя бы один представитель каждой из пятерок? Например, если все пятерки совпадают, то, очевидно, рассадка возможна. Но ведь есть огромное количество других вариантов! Может, если пятерки распределятся более хитро, то окажется, что, как ни рассаживай 30 школьников по двум кабинетам, обязательно найдется кабинет, в котором одна из пятерок находится целиком?

Ответ на вопрос все-таки положительный: да, такая рассадка всегда возможна. Решение очень простое и красивое! Оно основано на вероятностном методе в комбинаторике (см. [1], [2]). В принципе здесь еще можно было бы обойтись без ссылок на теорию вероятностей, но будет лучше, если мы сразу воспользуемся именно вероятностной терминологией.

Итак, нам даны 15 пятерок. Обозначим их $M_{1}, \ldots, M_{15}$. Рассмотрим случайную рассадку школьников. Что это значит? Вообще говоря, что угодно, ведь случайность можно определять по-разному. Но мы будем понимать случайность максимально просто: каждый школьник отправляется в первый кабинет с вероятностью $\frac{1}{2}$, и с такой же вероятностью он идет во второй кабинет; выбор кабинета школьни-

DOI: https://doi.org/10.4213/kvant20190802 ки осуществляют независимо друг от друга. Иными словами, мы как бы 30 раз бросаем симметричную монетку, и если монетка в $i$-м бросании падает решкой кверху, то $i$-й школьник идет в первый кабинет, иначе - во второй. Введем обозначение $A_{i}, i=1, \ldots, 15$, для события, состоящего в том, что пятерка $M_{i}$ целиком попала в один кабинет. Какова вероятность $\mathrm{P}\left(A_{i}\right)$ этого события? Поскольку выбор производится школьниками независимо, вероятности перемножаются, и мы получаем

$$
\mathrm{P}\left(A_{i}\right)=\left(\frac{1}{2}\right)^{5}+\left(\frac{1}{2}\right)^{5}=\frac{1}{16} .
$$

Теперь изучим вероятность того, что хотя бы одна пятерка целиком сидит в одном кабинете. Разумеется, это вероятность со$\begin{aligned} \text { бытия } & \bigcup_{i=1}^{15} A_{i} \text {. Ясно, что } \\ & \mathrm{P}\left(\bigcup_{i=1}^{15} A_{i}\right) \leq \sum_{i=1}^{15} \mathrm{P}\left(A_{i}\right)=\frac{15}{16}<1 .\end{aligned}$

Значит, с положительной вероятностью имеет место противоположное событие, которое состоит в том, что ни одна пятерка целиком не сидит в одном из кабинетов. Но ведь это ровно то, что нужно! «Ни одна пятерка целиком не сидит» - это то же самое, что «в каждом кабинете есть хотя бы один представитель каждой из пятерок». И это выполнено с положительной вероятностью. Если вероятность рассадки с нужным свойством положительна, то такая рассадка точно есть. Как ее искать другой вопрос. Но задача решена: рассадка есть всегда.

Отметим, что в нашем решении нигде не использовался тот факт, что всего школьников именно 30. Соответственно, возникает вопрос: а можно ли, по-прежнему пренебрегая исходным количеством школьников, увеличить количество предметов от 
15 и получить тот же результат с возможностью рассадки? До шестнадцати дотянуть легко. В самом деле, все события $A_{i}$ имеют непустое пересечение. Заведомо в этом пересечении находится дурацкая рассадка, при которой все школьники сидят в первом кабинете. Но тогда вероятность объединения строго менъше суммы вероятностей, и снова наш метод срабатывает. Уже с семнадцатью такой номер не проходит...

Легко видеть, что для 126 пятерок ответ уже положительным не является. Странное число, да? Ну, сейчас разберемся! Мы же вольны в выборе исходного количества школьников. Давайте возьмем все пятерки, какие только можно составить из девя$m и$ человек. Их в аккурат $126=C_{9}^{5}$. Попробуем теперь рассадить девятерых школьников по двум кабинетам. И вот не тут-то было! При любой рассадке в какойто кабинет попадет не менее пяти школьников. Но у нас каждая пятерка сейчас «в деле». Значит, все плохо, и мы имеем пример ситуации, когда ответ на первоначальный вопрос уже не является утвердительным.

Итак, для любых 16 пятерок ответ утвердительный, но существуют 126 пятерок, для которых ответ отрицательный. Все это приводит к общей постановке задачи, о которой мы и поговорим в следующем разделе.

\section{Постановка общей задачи}

Прежде всего, пора перейти от школьников к абстрактным объектам. А именно, введем понятие гиперграфа. Оно является прямым обобщением понятия графа. У гиперграфа также есть вершины, образующие некоторое конечное множество $V$, и ребра, образующие множество $E$. Только у гиперграфа в каждом ребре не обязательно две вершины: может быть и больше (ребра из одной вершины мы исключим из рассмотрения вовсе). Более строго, гиперграф - это пара $H=(V, E)$, где $V$ некоторое множество, а $E-$ некоторая совокупность подмножеств множества $V$. Подмножества неупорядоченные (сочетания без повторений), кратных ребер нет, в каждом ребре хотя бы две вершины. Гиперграф называется $n$-однородным, если в каждом его ребре ровно $n$ вершин. Обыкновенный граф, тем самым, - это 2-однородный гиперграф. А школьники и пятерки - это 5-однородный гиперграф на тридцати вершинах.

Часто замечают, что у гиперграфа, в отличие от графа, нет естественного «портрета». Действительно, если граф легко изобразить как множество точек на плоскости, соединенных отрезками (или дугами), то попытка сделать то же самое с гиперграфом приводит к странному хитросплетению эдаких «сарделек» (рис.1).

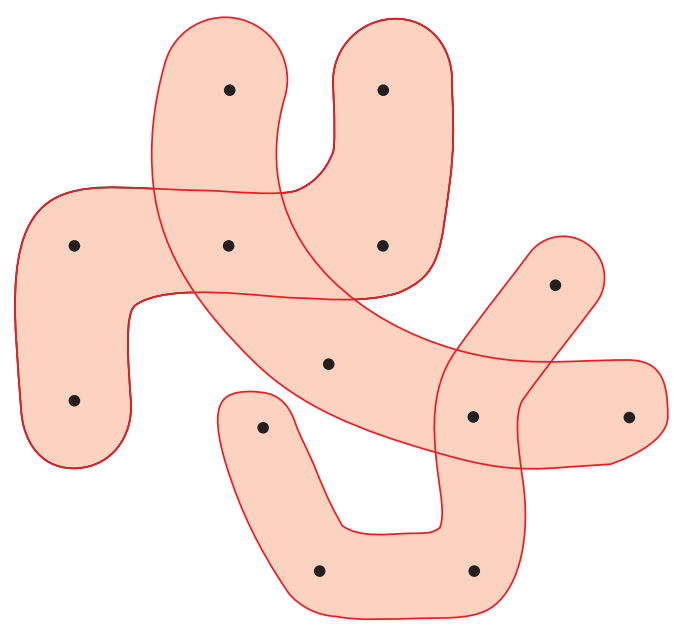

Pис. 1

В своих лекциях я часто употребляю слово «сарделька» для обозначения ребра гиперграфа, но картинок не рисую. Мне самому легче представлять себе именно эдакую кастрюльку (множество вершин) с намешанными в ней сардельками-ребрами. Некоторые «продвинутые пользователи» вспоминают выражение «симплициальный комплекс» (просьба тех, кто не слышал его, не пугаться, так как мы его употреблять не будем). Но (опять же, для тех, кто в теме) симплициальный комплекс - это гиперграф, у которого каждое подмножество каждого ребра само является ребром, т.е. это заведомо не однородный гиперграф. В общем, нам это знание не поможет, и мы больше о симплициальных комплексах вспоминать не будем. 
Назовем хроматическим числом гиперграфа $H$ величину $\chi(H)$, равную наименьшему количеству цветов, в которые можно так покрасить все вершины гиперграфа, чтобы каждое его ребро было неодноцветным. Позвольте, но ведь задача из предыдущего раздела отлично формулируется в этих терминах! В самом деле, вопрос о пятнадцати пятерках отныне звучит так: «Верно ли, что у любого 5-однородного гиперграфа с тридцатью вершинами и пятнадцатью ребрами хроматическое число равно двум?» Рассадка по двум кабинетам и раскраска в два цвета - лишь два способа описания одного и того же явления.

Введем, наконец, классическую величину $m(n)$, предложенную Эрдёшем и Хайналом в 1961 году и равную наименьшему $m$, при котором существует $n$-однородный гиперграф $H$ с $m$ ребрами и с $\chi(H)>2$. Главное сразу понять, что в терминах этой величины результаты предыдущего раздела выглядят так:

$$
17 \leq m(5) \leq 126 .
$$

У любого 5-однородного гиперграфа с шестнадцатью ребрами хроматическое число равно двум, поэтому $m(5) \geq 17$, но существует 5-однородный гиперграф со 126-ю ребрами, у которого хроматическое число больше двух, откуда $m(5) \leq 126$.

Очевидное обобщение неравенства (1) приводим ниже:

$$
2^{n-1}+1 \leq m(n) \leq C_{2 n-1}^{n} .
$$

Мы не станем доказывать эти неравенства, ведь это совсем легкое упражнение. Но мы обсудим вопрос о том, насколько близки друг к дружке верхняя и нижняя оценки. В самом деле, хорошо известно тождество (см. [3])

$$
\begin{aligned}
C_{2 n-1}^{0}+C_{2 n-1}^{1}+\ldots & +C_{2 n-1}^{n-1}+ \\
& +C_{2 n-1}^{n}+\ldots+C_{2 n-1}^{2 n-1}=2^{2 n-1} .
\end{aligned}
$$

Также известно, конечно, что два центральных слагаемых в этом тождестве являются в нем самыми большими. При этом общее число слагаемых в левой части тождества равно $2 n$. Следовательно,

$$
\frac{2^{2 n-1}}{2 n}<C_{2 n-1}^{n}<2^{2 n-1} \text {. }
$$

В итоге понятно, что зазор между оценками в (2) экспоненциальный: нижняя имеет порядок $2^{n}$, а верхняя с точностью до возможного деления на что-то порядка $n$ имеет порядок $4^{n}$. Это не очень круто, и это одна из серьезнейших проблем современной экстремальной комбинаторики! ( Раздел науки, с которым мы сейчас имеем дело, называется «экстремальной комбинаторикой» не потому, что только экстремалы им занимаются, хотя он и захватывающе красив и не менее захватывающе труден, но потому, что в его рамках ищутся экстремальные, т.е. максимальные или минимальные комбинаторные величины, среди которых $m(n)$. )

В последующих разделах мы изучим красивейшие подходы к уменьшению зазора в (2). Отметим, что нижняя оценка в (2) вероятностная (т.е. она гарантирует наличие раскраски, но не объясняет, как ее искать), а верхняя оценка конструктивная (приводится явный пример гиперграфа, не имеющего раскраски в 2 цвета). В следующем разделе мы значительно улучшим верхнюю оценку, но сделаем это... вероятностным методом, т.е. за улучшение мы заплатим потерей конструктивности.

Напоследок предлагаем читателю самостоятельно ответить на вопрос, чему равно $m(2)$ (совсем просто) и $m(3)$ (сложнее, но посильно). Отметим при этом, что $m(4)$ нашли совсем недавно с помощью весьма нетривиального компьютерного перебора, а величину $m(5)$, которая послужила нам в качестве затравки, никто до сих пор не знает!

\section{Верхняя оценка величины $\boldsymbol{m}(\boldsymbol{n})$}

В этом разделе мы докажем следующую теорему.

Теорема 1. Пусть дано число п. Пусть $v$ - любое четное число, не меньшее $2 n$. Положим

$$
p=\frac{2 C_{v / 2}^{n}}{C_{v}^{n}} .
$$

Пусть $m$ - наименьшее натуральное число, с которым выполнено неравенство

$$
2^{v}(1-p)^{m}<1
$$

Тогда $m(n) \leq m$. 
Для получения оптимальной оценки в теореме 1 нужно просто перебрать последовательно несколько первых $v$, начиная с $v=2 n$, и для каждого из них найти свое значение $m$. Наименьшее среди этих значений и будет оптимальным. Ниже мы приводим примеры подобных вычислений для некоторых $n$, попутно сравнивая полученные результаты с оценкой $m(n) \leq C_{2 n-1}^{n}$. Как видно, при малых $n$ теорема 1 дает более слабый результат. Однако чем больше $n$, тем яснее, что в теореме 1 оптимум ведет себя с точностью до некоторого постоянного множителя как $n^{2} \cdot 2^{n}$, и это уже грандиозно, ведь тем самым получается, что мы снижаем зазор, заменяя экспоненту от $n$ всего лишь квадратом этого числа! Константу можно сосчитать методами математического анализа, но мы не станем здесь этого делать.

На рисунке 2 показано сравнение верхних и нижних оценок для величины $m(n)$,

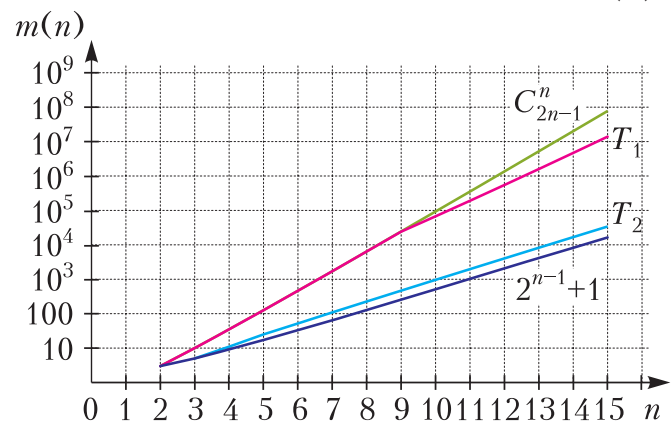
Pис. 2

данных в неравенстве (2) и теоремах 1 и 2. Обратите внимание: ось ординат логарифмическая, и поэтому то, что на графике похоже на линейную функцию, на самом деле растет экспоненцинально.

Для понимания доказательства теоремы 1 потребуется, помимо базовой вероятности, с которой мы уже немного свыклись, знание одного комбинаторного неравенства.

Неравенство выпуклости. Пусть $n \in \mathbb{N}$. Пусть $v \geq n-$ четное число. Пусть $a$, $b \in \mathbb{N}$ u $a+b=$ v. Тогдa

$$
\frac{C_{a}^{n}+C_{b}^{n}}{2} \geq C_{v / 2}^{n}=C_{(a+b) / 2}^{n} .
$$

Название неравенства происходит оттого, что оно говорит о выпуклости биноми- ального коэффициента как функции от нижнего индекса при заданном верхнем индексе. Конечно, четность $v$ не обязательна. Но так проще для восприятия. Неравенство очень простое, и мы оставляем его доказательство читателю. Заметим, что мы считаем $C_{m}^{k}=0$ при $k>m$.

Доказательство теоремы 1. Построим случайный $n$-однородный гиперграф с $v$ вершинами и $m$ ребрами. Нам нужно будет так осуществить построение, чтобы с положительной вероятностью в любой раскраске вершин случайного гиперграфа в 2 цвета было хотя бы одно одноцветное ребро или, что равносильно, чтобы с вероятностью, меньшей единицы, нашлась раскраска вершин случайного гиперграфа в 2 цвета, при которой все ребра неодноцветны. Рассмотрим множество вершин $V=\{1, \ldots v\}$. Случайными будут ребра. Выберем каждое из них независимо ото всех остальных из множества всех $n$-сочетаний из $V$ с вероятностью $\frac{1}{C_{v}^{n}}$. Читатель может спросить: «А что, если появятся кратные ребра? Ведь при взаимно независимом выборе ребра могут и совпасть». Но ответ простой. Если какие-то ребра совпадут, мы их отождествим. Получится гиперграф с еще меньшим числом ребер, а нам это только на пользу, раз уж мы доказываем сейчас верхнюю оценку для $m(n)$. Итак, пусть $H=(V, E)$ - это описанный только что случайный гиперграф и $E=\left\{f_{1}, \ldots, f_{m}\right\}$.

Пусть $\chi$ - некоторая раскраска $V$ в два цвета. Пусть в ней $a$ красных и $b$ синих вершин. Естественно, $v=a+b$. Обозначим $A_{\chi, i}$ событие, состоящее в том, что ребро $f_{i}$ одноцветно в раскраске $\chi$. Очевидно,

$$
\mathrm{P}\left(A_{\chi, i}\right)=\frac{C_{a}^{n}+C_{b}^{n}}{C_{\tau}^{n}} .
$$

За счет неравенства выпуклости получаем оценку

$$
\mathrm{P}\left(A_{\chi, i}\right)=\frac{C_{a}^{n}+C_{b}^{n}}{C_{v}^{n}} \geq \frac{2 C_{\tau / 2}^{n}}{C_{v}^{n}}=p .
$$

Тогда вероятность отрицания события $A_{\chi, i}$ (ребро $f_{i}$ неодноцветно) не больше $1-p$. 
Пусть $A_{\chi}$ - событие, при котором все ребра случайного гиперграфа неодноцветны в раскраске $\chi$. Поскольку ребра выбирались независимо друг от друга, получаем неравенство

$$
\mathrm{P}\left(A_{\chi}\right) \leq(1-p)^{m} .
$$

Наконец, интересующее нас событие $A-$ «найдется раскраска вершин случайного гиперграфа в 2 цвета, при которой все ребра неодноцветны» - это $\bigcup_{\chi} A_{\chi}$. Значит, $\mathrm{P}(A) \leq \mathrm{P}\left(\bigcup_{\chi} A_{\chi}\right) \leq \sum_{\chi} \mathrm{P}\left(A_{\chi}\right) \leq 2^{v}(1-p)^{m}$, ведь всего раскрасок $V$ в 2 цвета $2^{v}$ штук. Последняя величина, как нам того и нужно, меньше единицы по условию теоремы, и теорема доказана.

Крайне любопытно, что, жертвуя конструктивностью, мы реально упростили себе жизнь. До сих пор не известны явные конструкции гиперграфов со столь малым числом ребер и хроматическим числом, большим двух. Лишь совсем недавно - в 2013 году - Гебауэр построила пример гиперграфа, у которого число ребер не превосходит величины

$$
2^{n+c n^{2 / 3}}, \quad c>0 .
$$

Это круто, поскольку основной сомножитель $-2^{n}-$ совпадает с известной нам экспонентой в верхней и нижней оценках. Но это гораздо слабее теоремы 1 , ведь $n^{2}$ несравнимо меньше, чем $2^{n^{2 / 3}}$.

\section{Улучшение нижней оценки величины $\boldsymbol{m}(\boldsymbol{n})$ с помощью жадного алгоритма}

В этом разделе мы улучшим известную нам нижнюю оценку $m(n)$.

Теорема 2. Пусть для данного п число $m$ является наибольшим натуральньм, с которым выполнено неравенство

$$
m^{2} \cdot \frac{((n-1) !)^{2}}{(2 n-1) !}<1
$$

Тогда

$$
m(n)>m .
$$

Как и в предыдущем разделе, выпишем ниже результат сопоставления нижней оценки в (2) и в (3). Здесь видно, что даже при $n=5$ есть заметное улучшение. Опять же, методы математического анализа позволяют показать, что оценка (3) растет как $c \cdot \sqrt[4]{n} \cdot 2^{n}$ с некоторой фиксированной $c>0$. Мы здесь в такие детали не влезаем.

Доказательство теоремы 2 основано на красивой и несложной идее, которую предложил Плухар. Для описания идеи введем несколько новых понятий. Пусть дан гиперграф $H=(V, E)$. Изначально его вершины представляют собой некоторую совокупность объектов, которые никак не упорядочены. Порядок на этой совокупности можно задать, разумеется, $|V|$ ! способами. Пусть задан некоторый порядок (нумерация вершин) $\pi$ и есть два ребра $f_{1}, f_{2}$, имеющие ровно одну общую вершину $i$. Назовем пару $\left(f_{1}, f_{2}\right)$ упорядоченной 2 -цепью в нумерации $\pi$, если номера всех вершин ребра $f_{1}$ предшествуют номеру $i$, а номера всех вершин ребра $f_{2}$ идут после номера вершины $i$. На рисунке 3 показана «кастрюлька с двумя сардельками» и два

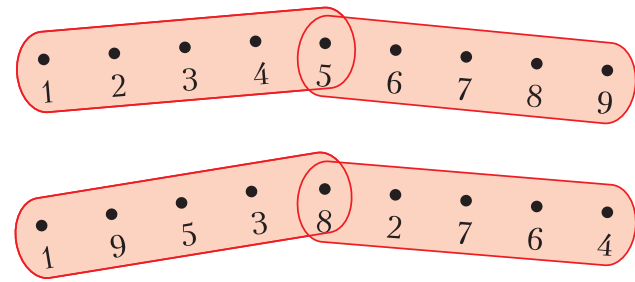

Pис. 3

способа нумерации вершин в кастрюльке, при одном из которых сардельки образуют упорядоченную 2-цепь, а при другом - не образуют. Идея Плухара формулируется в виде следующего критерия.

Критерий Плухара. Хроматическое число гиперграфа равно двум тогда и только тогда, когда существует нумерачия его вершин, в которой нет упорядоченных 2-иепей.

Прежде чем привести простое доказательство критерия, полезно осознать, что он говорит в случае обыкновенного графа (т.е. 2-однородного гиперграфа). В самом деле, что означает утверждение «хроматическое число графа равно двум»? Оно означает, что множество вершин графа можно разделить на две непересекающиеся части, внутри которых ребер графа нет, но между которыми как раз и прохо- 


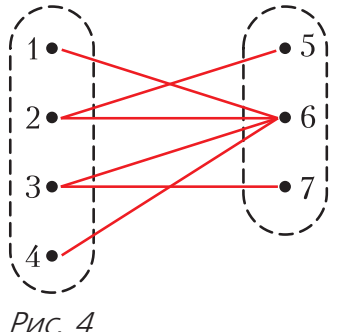

Рис. 4 дят все ребра графа (рис.4). Такой граф еще называют двудольным (т.е. буквально двухчастным), и многие читатели наверняка сталкивались с этим объектом. Понятно, что вершинам одной доли можно присвоить номера от единицы до числа, равного количеству вершин в этой доле, а вершинам второй доли - все последующие номера, и тогда упорядоченных 2-цепей не возникнет (здесь 2-цепь это «галочка», у которой номера вершин идут в порядке «меньше-больше-меньше» или «больше-меньше-больше»).

Доказательство критерия Плухара. В одну сторону мы фактически доказательство уже привели. Действительно, если существует двухцветная раскраска, при которой все ребра неодноцветны, то достаточно взять любую нумерацию, при которой все вершины первого цвета имеют меньшие номера, нежели все вершины второго цвета (двудольность).

В обратную сторону рассуждение основано на простейшем жадном алгоритме, и это объясняет название раздела. Итак, пусть существует нумерация вершин без упорядоченных 2-цепей. Обозначим будущие цвета числами $1,2, \ldots$ Рассматриваем вершины по порядку и красим их в минимальный цвет, с которым они не образуют одноцветных ребер вместе с уже покрашенными вершинами. Если для некоторой вершины $v$ нам не хватает цветов 1 и 2, то существует ребро $f_{2}$, которому принадлежит вершина $v$ и в котором все остальные (предшествующие) вершины уже покрашены в цвет 2. Пусть $ш-$ вершина в $f_{2}$ с наименьшим номером. Раз мы ее покрасили в свое время в цвет 2, то мы не смогли тогда ее покрасить в цвет 1. Почему? А потому, что, стало быть, имелось ребро $f_{1}$, для которого вершина ж была, наоборот, последней и которое имело все вершины цвета 1. В этом случае очевидно, что ребра $f_{1}, f_{2}$ образуют упорядоченную 2-цепь. Противоречие.
Критерий полностью доказан.

Доказательство теоремы 2. Пусть дан произвольный $n$-однородный гиперграф $H=(V, E)$ с $m$ ребрами. Мы хотим показать, что $\chi(H)=2$. Согласно критерию Плухара достаточно доказать существование нумерации $V$ без упорядоченных 2цепей. Рассмотрим, как водится, случайную нумерацию. Иными словами, каждая нумерация выбирается с вероятностью $\frac{1}{|V| !}$. Пусть $f_{1}, f_{2} \in E$ и пересечение этих ребер состоит из одной вершины. Обозначим $A_{f_{1}, f_{2}}$ событие, состоящее в том, что в случайной нумерации ребра $f_{1}, f_{2}$ образуют упорядоченную 2-цепь. Легко сообразить, что

$$
P\left(A_{f_{1}, f_{2}}\right)=\frac{((n-1) !)^{2}}{(2 n-1) !} .
$$

Далее, вероятность того, что найдутся два ребра, образующие упорядоченную 2-цепь, равна

$$
\begin{aligned}
& \mathrm{P}\left(\bigcup_{f_{1}, f_{2}} A_{f_{1}, f_{2}}\right) \leq \sum_{f_{1}, f_{2}} \mathrm{P}\left(A_{f_{1}, f_{2}}\right)< \\
& \quad<|E|^{2} \cdot \frac{((n-1) !)^{2}}{(2 n-1) !}=m^{2} \cdot \frac{((n-1) !)^{2}}{(2 n-1) !}<1,
\end{aligned}
$$

и теорема доказана.

\section{Дальнейшее улучшение нижней оценки $\boldsymbol{m}(\boldsymbol{n})$ с помощью рандомизированного алгоритма перекраски}

В этом разделе мы используем иной вероятностный подход, нежели до сих пор, и докажем следующую теорему, придуманную Беком и Спенсером.

Теорема 3. Пусть дано п и $x$-максимальное число, для которого существует $p \in[0,1]$, удовлетворяющее неравенству

$$
x \cdot(1-p)^{n}+x \cdot p^{n}+x^{2} \cdot p \cdot(1+p)^{n}<1 .
$$

Тогда $m(n) \geq x \cdot 2^{n-1}$.

Опять-таки, сравним теорему 3 с теоремой 2 , приводя ниже результаты компьютерных вычислений. Аналитические методы позволяют показать, что здесь оптимальное значение $x$ - это $c \cdot \sqrt[3]{\frac{n}{\ln n}}$, но мы не хотим здесь требовать от читателя даже 
знания того, что такое $\ln n$. В любом случае оценка еще круче прежней.

Доказательство теоремы 3. Пусть $H=(V, E)-n$-однородный гиперграф с

$$
m=\left[x \cdot 2^{n-1}\right]
$$

ребрами. Покажем, что существует раскраска в 2 цвета множества $V$, при которой все ребра неодноцветны. Раскраску будем строить с помощью следующего рандомизированного алгоритма.

Шаг 1. Красим вершины независимо друг от друга, с вероятностью $\frac{1}{2}$ выбирая для каждой вершины один из двух цветов - красный или синий. Пусть $D-$ случайное множество вершин, принадлежащих одноцветным ребрам (объединение всех ребер, которые оказались одноцветными). Это может быть и пустое множество, например.

Шаг 2. Рассматриваем только вершины из множества $D$. У каждой из них мы независимо ото всех остальных вершин множества $D$ меняем цвет на противоположный с вероятностью $p$ и не меняем цвет с вероятностью $1-p$. Иными словами, у нас как бы есть монета со смещенным, вообще говоря, центром тяжести. При случайном бросании монета ложится решкой кверху с вероятностью $p$ и орлом вероятностью $1-p$. Мы бросаем монету $|D|$ раз, и если в очередном бросании монета падает решкой кверху, то меняем цвет соответствующей вершины из $D$; иначе не меняем.

Понятно, что шаг 1 - это обычная случайная раскраска, с помощью которой мы доказывали неравенство $m(n) \geq 2^{n-1}+1$. Таким образом, шаг 2 - это попытка исправить ошибки шага 1 за счет того, что шаг 1 не чувствителен к виду исходного гиперграфа, а шаг 2 пытается учесть его структуру и повысить, тем самым, вероятность того, что на выходе все ребра окажутся неодноцветными.

Пусть $\mathcal{F}$ - событие, состоящее в том, что раскраска не удалась, т.е. существуют одноцветные ребра. Как конкретное ребро $f$ может оказаться одноцветным? Есть всего 6 вариантов:
1. $A_{f, 1}$ : ребро $f$ красное после шага 1 и красное после шага 2 ;

2. $A_{f, 2}$ : ребро $f$ красное после шага 1 и синее после шага 2;

3. $A_{f, 3}$ : peбро $f$ синее после шага 1 и синее после шага 2 ;

4. $A_{f, 4}$ : ребро $f$ синее после шага 1 и красное после шага 2 ;

5. $C_{f, 1}$ : ребро $f$ неодноцветное после шага 1 и красное после шага 2 ;

6. $C_{f, 2}$ : ребро $f$ неодноцветное после шага 1 и синее после шага 2.

Очевидно,

$$
\begin{gathered}
\mathcal{F}= \\
=\bigcup_{f \in E}\left(A_{f, 1} \cup A_{f, 2} \cup A_{f, 3} \cup A_{f, 4} \cup C_{f, 1} \cup C_{f, 2}\right), \\
\mathrm{P}\left(A_{f, 1}\right)=\mathrm{P}\left(A_{f, 3}\right), \mathrm{P}\left(A_{f, 2}\right)=\mathrm{P}\left(A_{f, 4}\right), \\
\mathrm{P}\left(C_{f, 1}\right)=\mathrm{P}\left(C_{f, 2}\right) .
\end{gathered}
$$

Поэтому

$$
\mathrm{P}(\mathcal{F}) \leq 2 \sum_{f \in E}\left(\mathrm{P}\left(A_{f, 1}\right)+\mathrm{P}\left(A_{f, 2}\right)+\mathrm{P}\left(C_{f, 1}\right)\right) .
$$

Оценим вероятности, стоящие в скобках под знаком суммирования. Совсем легко разобраться с первыми двумя:

$$
\mathrm{P}\left(A_{f, 1}\right)=2^{-n} \cdot(1-p)^{n}, \mathrm{P}\left(A_{f, 2}\right)=2^{-n} \cdot p^{n} .
$$

А вот с третьей вероятностью намного труднее.

Как могло случиться, что ребро $f$ неодноцветное после шага 1, но красное после шага 2? Конечно, синие вершины ребра $f$, имевшиеся в нем после шага 1, должны были перекраситься. Но почему? Как они попали в множество $D$, если $f$ неодноцветное и в формировании $D$ не участвовало? Значит, было еще хотя бы одно ребро $\varphi$, которое было синим после шага 1 и которое имеет непустое пересечение с $f$. Это именно следствие, не равносильность! Все могло быть очень и очень хитро. Например, красные после шага 1 вершины ребра $f$ тоже могли попасть в $D$ и пытаться поменять цвет, но монетка легла орлом. И так далее. Но мы точно знаем, что следствие имеет место, откуда

$$
\mathrm{P}\left(C_{f, 1}\right) \leq \mathrm{P}\left(\bigcup_{\varphi} B_{f, \varphi}\right),
$$


где объединение берется по всем $\varphi \in E$, которые имеют непустое пересечение с $f$, a $B_{f, \varphi}$ - событие, состоящее в том, что $f$ неодноцветное после шага $1, f$ красное после шага 2 и $\varphi$ синее после шага 1. Таким образом,

$$
\mathrm{P}\left(C_{f, 1}\right) \leq \sum_{\varphi} \mathrm{P}\left(B_{f, \varphi}\right),
$$

и нам нужно оценить величину $\left.\mathrm{P}\left(B_{f, \varphi}\right)\right)$.

Положим $h=|f \cap \varphi| \geq 1$. Посмотрим отдельно на $a=f \cap \varphi$, отдельно на $b=\varphi \backslash f$ и отдельно на $c=f \backslash \varphi$. С вершинами из $a$ все ясно. Они были синими и стали красными. Вероятность этого $2^{-h} \cdot p^{h}$. Так же просто все и в случае $c$. Вершины там были синими, а что с ними стало, мы не знаем. Вероятность этого не больше, чем $2^{-(n-h)} \cdot 1$. Интереснее всего обстоят дела с b. Пусть $v \in b$. Есть два варианта. Вопервых, $v$ могла быть синей и стать красной. Вероятность этого $\frac{1}{2} \cdot p$. Во-вторых, она могла быть красной и остаться красной. Как произошло последнее, мы не знаем: то ли $v$ попала в $D$, но монетка легла орлом, то ли $v$ не попала в $D$ и просто не пыталась сменить цвет. В любом случае здесь вероятность не больше, чем $\frac{1}{2} \cdot 1$. Итого для данной $v \in b$ имеем оценку вероятности величиной, равной сумме оценок вероятностей первого и второго варианта, т.е. $\frac{p}{2}+\frac{1}{2}$. В целом по $b$ за счет независимости вероятность оценивается величиной $\left(\frac{p}{2}+\frac{1}{2}\right)^{n-h}$. Собирая все оценки вместе, получаем

$$
\begin{aligned}
\mathrm{P}\left(B_{f, \varphi}\right) \leq 2^{-h} \cdot p^{h} \cdot 2^{-(n-h)} \cdot\left(\frac{p}{2}+\frac{1}{2}\right)^{n-h}= \\
=2^{h-2 n} \cdot p^{h} \cdot(1+p)^{n-h} .
\end{aligned}
$$

Очевидно, последняя величина принимает максимальное значение при $h=1$ (она убывает по $h)$. Значит, всегда

$$
\begin{aligned}
\mathrm{P}\left(B_{f, \varphi}\right) \leq 2^{1-2 n} \cdot p \cdot(1+p)^{n-1}< & \\
& <2^{1-2 n} \cdot p \cdot(1+p)^{n} .
\end{aligned}
$$

Вернемся к оценке вероятности $C_{f, 1}$. Ясно, что

$$
\begin{aligned}
& \mathrm{P}\left(C_{f, 1}\right) \leq \sum_{\varphi} \mathrm{P}\left(B_{f, \varphi}\right)<|E| \cdot 2^{1-2 n} \cdot p \cdot(1+p)^{n}= \\
&=m \cdot 2^{1-2 n} \cdot p \cdot(1+p)^{n} \leq \\
& \leq x \cdot 2^{n-1} \cdot 2^{1-2 n} \cdot p \cdot(1+p)^{n}= \\
&=x \cdot 2^{-n} \cdot p \cdot(1+p)^{n} .
\end{aligned}
$$

Далее,

$$
\begin{array}{rl}
\mathrm{P}(\mathcal{F}) \leq 2 \sum_{f \in E}\left(\mathrm{P}\left(A_{f, 1}\right)+\mathrm{P}\left(A_{f, 2}\right)+\mathrm{P}\left(C_{f, 1}\right)\right) \leq \\
\leq 2 \sum_{f \in E}\left(2^{-n} \cdot(1-p)^{n}+2^{-n} \cdot p^{n}+\right. \\
\left.\quad+x \cdot 2^{-n} \cdot p \cdot(1+p)^{n}\right)= \\
=2 & m\left(2^{-n} \cdot(1-p)^{n}+2^{-n} \cdot p^{n}+\right. \\
\quad & \left.\quad x \cdot 2^{-n} \cdot p \cdot(1+p)^{n}\right) \leq \\
\leq \quad x & \cdot 2^{n}\left(2^{-n} \cdot(1-p)^{n}+2^{-n} \cdot p^{n}+\right. \\
& \left.+x \cdot 2^{-n} \cdot p \cdot(1+p)^{n}\right)= \\
= & x \cdot(1-p)^{n}+x \cdot p^{n}+x^{2} \cdot p \cdot(1+p)^{n} .
\end{array}
$$

Последняя величина меньше единицы по условию теоремы, и теорема доказана.

Заметим, что сейчас самые лучшие известные оценки при больших $n$ имеют вид

$$
c_{1} \cdot \sqrt{\frac{n}{\ln n}} \cdot 2^{n} \leq m(n) \leq c_{2} \cdot n^{2} \cdot 2^{n} .
$$

Таким образом, полученная нами верхняя оценка фактически лучшая из известных! А нижняя оценка отличается от самой лучшей лишь видом корня, что тоже уже близко к рекорду.

Пока нет даже правдоподобной гипотезы о том, как ведет себе на самом деле величина $m(n)$ ! Возможно, читатель решит когда-нибудь эту проблему?

\section{Литература}

1. Н.Алон, Дж.Спенсер. Вероятностный метод. - М.: Бином. Лаборатория знаний, 2007.

2. А.М.Райгородский. Вероятность и алгебра в комбинаторике. - М.: МЦНМО, 2015.

3. Н.Я.Виленкин. Комбинаторика. - М.: Наука. Главная редакция физико-математической литературы, 1969. 